\title{
DESIGN OF ROBUST EXPLICIT MODEL PREDICTIVE CONTROLLER VIA ORTHOGONAL SEARCH TREE PARTITIONING
}

\author{
A. Grancharova, T. A. Johansen \\ Department of Engineering Cybernetics, Norwegian University of Science and Technology, 7491 Trondheim, Norway \\ E-mail: Alexandra.Grancharova@itk.ntnu.no, Tor.Arne.Johansen@itk.ntnu.no, Fax: +47 73594399
}

Keywords: Model predictive control, Multi-parametric quadratic programming, Robust control.

\begin{abstract}
Explicit piecewise linear state feedback solutions to the constrained linear model predictive control problem have been characterized and computed using multi-parametric quadratic programming. The piecewise linear state feedback is defined on a polyhedral partition of the state space, which may be quite complex. Recently, approximate multiparametric quadratic programming approaches have been developed, which have the advantage that the state space partition is structured as an orthogonal search tree. This leads to more efficient real-time computations and admits implementation at high sampling frequencies in embedded systems with inexpensive processors and low software complexity. This paper presents an approximate multiparametric quadratic programming algorithm that allows the explicit solution of robust model predictive control problems, by imposing an orthogonal search tree structure on the partition. Here, the robustness is defined in terms of satisfaction of the input and output constraints under all possible disturbance realizations.
\end{abstract}

\section{Introduction}

Model predictive control (MPC) is an efficient methodology to solve complex constrained multivariable control problems. The requirement to perform on-line optimization however limits the applicability of MPC mostly to slowly varying processes. Recently, several methods for explicit solution of MPC problems have been developed. The main motivation behind explicit MPC is that an explicit state feedback law avoids the need for real-time optimization, and is therefore potentially useful for applications with fast sampling where MPC has not traditionally been used. In [1] it was recognized that the constrained linear MPC problem is a multi-parametric quadratic program (mp-QP), when the state is viewed as a parameter to the problem. It was shown that the solution (the control input) has an explicit representation as a piecewise linear (PWL) state feedback on a polyhedral partition of the state space, see also $[2,8,14,15]$, and they develop an mp-QP algorithm to compute a representation of this function. Some of these approaches have been further extended to ensure robustness of the explicit MPC controllers against disturbances [3,11,13]. In [11] it is assumed that the disturbance input belongs to a compact polyhedral set, and the approach in [7] is applied to ensure feasible operation of the MPC controller that minimizes the nominal value of the performance index. This work has been further extended to proportional integral controllers [13]. In [3], an approach to explicit solution of robust MPC problems based on a minmax formulation with a performance index expressed in $\infty$ norm has been proposed. In [12], a min-max strategy for design of robust optimal controllers for piecewise affine systems with bounded disturbances is formulated and implemented by applying the dynamic programming technique. It has to be mentioned however, that solution obtained by optimizing the worst value of the performance criterion can be quite conservative.

Recently, in $[6,9,10]$, algorithms that determine an approximate explicit PWL state feedback solution by imposing an orthogonal search tree structure on the partition, have been developed. They may lead to more efficient realtime computations and admit implementation at high sampling frequencies in embedded systems with inexpensive processors and low software complexity. This paper suggests an approach to explicit solution of constrained linear MPC problems in the presence of bounded disturbances and represents an extension of the approximate mp-QP approach [10]. Like in [11], the explicit MPC controller minimizes the nominal value of the performance index and it is robust in the sense that all constraints are satisfied for all possible disturbance realizations within the specified range. However, here we consider a special case where the set of the disturbance inputs represents a hyper-rectangle that includes the origin in its interior and the constraints are in the form of upper and lower bounds on the input and output variables. Based on these assumptions, the conditions which guarantee feasible operation of the MPC controller are derived and the original mp-QP problem with disturbance input is converted into an mp-QP problem without disturbances. 


\section{Problem formulation}

Consider the linear discrete-time system:

$$
\begin{aligned}
& x(t+1)=A x(t)+B u(t)+T \theta(t) \\
& y(t)=C x(t)
\end{aligned}
$$

where $\boldsymbol{x}(\boldsymbol{t}) \in \boldsymbol{R}^{n}, \boldsymbol{u}(\boldsymbol{t}) \in \boldsymbol{R}^{m}$, and $\boldsymbol{y}(\boldsymbol{t}) \in \boldsymbol{R}^{p}$ are the state, input and output variable, $\theta(t)$ is the disturbance input that is assumed to belong to a bounded polyhedral set $\theta(t) \in \Theta^{A} \subset \boldsymbol{R}^{s}$. Also, $A \in \boldsymbol{R}^{n \times n}, \quad B \in \boldsymbol{R}^{n \times m}, \quad \boldsymbol{C} \in \boldsymbol{R}^{p \times n}$, $\boldsymbol{T} \in \boldsymbol{R}^{n \times s}$ and $(\boldsymbol{A}, \boldsymbol{B}) \quad$ is a controllable pair. Let $\Theta \equiv\left[\theta_{t}^{T}, \ldots, \theta_{t+N-1}^{T}\right]^{T} \in \Theta^{B}$ is a disturbance realization, with $\Theta \in \Theta^{B}=\left\{\Theta^{A} \times \Theta^{A} \ldots \times \Theta^{A}\right\} \subset \boldsymbol{R}^{s N}$. It is assumed that a full measurement of the state $\boldsymbol{x}(\boldsymbol{t})$ is available at the current time $\boldsymbol{t}$. Then, for the current $\boldsymbol{x}(\boldsymbol{t})$, MPC solves the optimization problem:

$$
V^{*}(x(t), \Theta)=\min _{U \equiv\left\{u_{t}, \ldots, u_{t+N-1}\right\}} J(U, x(t), \Theta)
$$

subject to:

$$
\begin{aligned}
& y_{\min } \leq y_{t+k \mid t} \leq y_{\max }, k=1, \ldots, N \\
& u_{\min } \leq u_{t+k} \leq u_{\max }, k=0,1, \ldots, N-1 \\
& x_{t \mid t}=x(t) \\
& x_{t+k+1 \mid t}=A x_{t+k \mid t}+B u_{t+k}+T \theta_{t+k}, \theta_{t+k} \in \Theta^{A}, k \geq 0 \\
& y_{t+k \mid t}=C x_{t+k \mid t}, k \geq 0
\end{aligned}
$$

with the cost function given by:

$$
\begin{gathered}
J(U, x(t), \Theta)=\sum_{k=0}^{N-1}\left[x_{t+k \mid t}^{T} Q x_{t+k \mid t}+u_{t+k}^{T} R u_{t+k}\right] \\
+x_{t+N \mid t}^{T} P x_{t+N \mid t}
\end{gathered}
$$

and symmetric $\boldsymbol{R}>0, \boldsymbol{Q} \geq 0, \boldsymbol{P}>0$. The final cost matrix $\boldsymbol{P}$ may be taken as the solution of the algebraic Riccati equation. It is also assumed $\boldsymbol{u}_{\text {max }}>0>\boldsymbol{u}_{\min }, \boldsymbol{y}_{\max }>0>\boldsymbol{y}_{\min }$, such that the origin is an interior point in the feasible set $X_{f}=\left\{\boldsymbol{x}(\boldsymbol{t}) \in \boldsymbol{R}^{n} \mid \exists \boldsymbol{U}\right.$ satisfying (3)-(7) $\}$. Here, we consider the nominal optimization criterion:

$$
V_{\text {nom }}^{*}(x(t))=\min _{U \equiv\left\{u_{t}, \ldots, u_{t+N-1}\right\}} J\left(U, x(t), \theta^{N}\right)
$$

corresponding to $\theta(t)=\theta^{N}=0$, where $\theta^{N}$ is the nominal value of the disturbance input.

In this problem formulation, the robustness is defined in terms of satisfaction of the output and input constraints (3) and (4) under all possible disturbance realizations $\Theta \in \Theta^{B}$ that influence the state of the system (Equation (6)).

By substituting:

$$
\boldsymbol{x}_{t+k \mid t}=A^{k} \boldsymbol{x}(\boldsymbol{t})+\sum_{j=0}^{k-1} A^{j} B \boldsymbol{u}_{t+k-1-j}+\sum_{j=0}^{k-1} A^{j} \boldsymbol{T} \theta_{t+k-1-j}
$$

in the constraints (3) - (7), they can be represented in the form:

$$
G U \leq W+E_{1} x(t)+E_{2} \Theta \quad, \forall \Theta \in \Theta^{B}
$$

where $\boldsymbol{U} \equiv\left[\boldsymbol{u}_{t}^{T}, \ldots, \boldsymbol{u}_{t+N-1}^{T}\right]^{T} \in \boldsymbol{R}^{m N}$ is the optimization vector and $\Theta \in \Theta^{B}$ is the disturbance realization. Then the nominal optimization criterion (9) is rewritten as:

$$
\begin{aligned}
V_{\text {nom }}^{*}(x(t))=\min _{U} & \left\{\frac{1}{2} U^{T} H U+x^{T}(t) F U\right\} \\
+ & \frac{1}{2} x^{T}(t) Y x(t)
\end{aligned}
$$

By defining:

$$
z \equiv U+H^{-1} F^{T} x(t),
$$

where $\boldsymbol{z} \in \boldsymbol{R}^{\boldsymbol{m} N}$, the optimization problem (12) subject to constraint (11), is transformed into the following mp-QP problem:

$$
V_{z, \text { nom }}^{*}(x)=\min _{z} \frac{1}{2} z^{T} H z
$$

subject to:

$$
G z \leq W+S_{1} x(t)+S_{2} \Theta \quad, \forall \Theta \in \Theta^{B}
$$

\section{Approximate mp-QP algorithm for design of robust explicit MPC}

\subsection{Feasibility in the presence of disturbance}

\section{Assumption 1:}

The disturbance input set:

$$
\Theta^{A}=\left\{\theta \in \boldsymbol{R}^{s} \mid \theta^{L} \leq \theta \leq \theta^{U}\right\}
$$

represents a hyper-rectangle that includes the origin in its interior.

\section{Definition 1:}

Consider the $i$-th constraint defined by $\boldsymbol{G}^{i}, \boldsymbol{W}^{i}, \boldsymbol{S}_{1}^{i}, \boldsymbol{S}_{2}^{i}$ rows of the matrices $\boldsymbol{G}, \boldsymbol{W}, \boldsymbol{S}_{1}, \boldsymbol{S}_{2}$. The worst disturbance realization for the $i$-th constraint, denoted by $\tilde{\Theta}^{i} \in \Theta^{B}$ is one which solves the linear program:

$$
S_{2}^{i} \tilde{\Theta}^{i}=\min _{\Theta \in \Theta^{B}}\left\{S_{2}^{i} \Theta\right\}
$$

\section{Lemma 1:}

If there exists a $z$ that satisfies the following constraint:

$$
G z \leq \tilde{W}+S_{1} x(t),
$$

where the $i$-th row of the matrix $\tilde{W}$ is determined by:

$$
\tilde{W}^{i}=\boldsymbol{W}^{i}+\boldsymbol{S}_{2}^{i} \tilde{\Theta}^{i},
$$

and where $\tilde{\Theta}^{i} \in \Theta^{B}$ is the worst disturbance realization for the $i$-th constraint, then this implies that $z$ will satisfy constraint (15) for all possible disturbance realizations $\Theta \in \Theta^{B}$. Such a $z$ is referred to as robustly feasible.

The proof of Lemma 1 is straightforward.

In this way, a constraint (18) which ensures robust feasibility can be easily constructed. Then, the original mp-QP problem (14) - (15) becomes:

$$
V_{z, \text { nom }}^{*}(x)=\min _{z} \frac{1}{2} z^{T} H z
$$

subject to: 


$$
G z \leq \tilde{W}+S_{1} x(t),
$$

where $\tilde{W}$ is determined by (19). Thus the original mp-QP problem with disturbance input (problem (14) - (15)) is reformulated as an mp-QP problem without disturbance (problem (20) - (21)) and therefore the existing approximate approach [10] for explicit solution of mp-QP problems can easily be applied to this problem. The optimal PWL solution to the problem (20) - (21) will be denoted $z^{*}(\boldsymbol{x})$. It has to be stressed that the approximate approach [10] guarantees that the optimal solution is feasible in sense that it will satisfy constraint (21). This directly implies by Lemma 1 above that constraint (15) of the original mp-QP problem will be satisfied for all possible disturbance realizations. This is summarized in the following Lemma:

Lemma 2 (feasible control in the presence of disturbance): Consider the bounded polyhedron $\boldsymbol{X}_{0}$ with vertices $\left\{\boldsymbol{v}_{1}, \boldsymbol{v}_{2}, \ldots, \boldsymbol{v}_{\boldsymbol{M}}\right\}$. If $\boldsymbol{K}_{0}$ and $\boldsymbol{g}_{0}$ solve the $Q P$ :

$$
\min _{\boldsymbol{K}_{0}, g_{0}} \sum_{i=1}^{M}\left(z^{*}\left(\boldsymbol{v}_{i}\right)-\boldsymbol{K}_{0} \boldsymbol{v}_{\boldsymbol{i}}-\boldsymbol{g}_{0}\right)^{T} \boldsymbol{H}\left(z^{*}\left(\boldsymbol{v}_{\boldsymbol{i}}\right)-\boldsymbol{K}_{0} \boldsymbol{v}_{\boldsymbol{i}}-\boldsymbol{g}_{0}\right)
$$

subject to:

$$
\boldsymbol{G}\left(\boldsymbol{K}_{0} \boldsymbol{v}_{i}+\boldsymbol{g}_{0}\right) \leq \tilde{\boldsymbol{W}}+\boldsymbol{S}_{1} \boldsymbol{v}_{i}, \boldsymbol{i} \in\{1,2, \ldots, M\},
$$

then the least squares approximation $\hat{z}_{0}(\boldsymbol{x})=\boldsymbol{K}_{0} \boldsymbol{x}+\boldsymbol{g}_{0}$ is robustly feasible for the $m p-Q P(14)-(15)$ for all $\boldsymbol{x} \in \boldsymbol{X}_{0}$ and all disturbance realizations $\Theta \in \Theta^{B}$.

Proof: It follows from Lemma 1 from [4] and from Lemma 1 given above.

\subsection{Approximate mp-QP algorithm}

Here an approximate $\mathrm{mp}-\mathrm{QP}$ algorithm is described to solve explicitly the mp-QP problem with disturbance input (problem (14) - (15)). We restrict our attention to a hyperrectangle $\boldsymbol{X} \subset \boldsymbol{R}^{n}$ where we seek to approximate the optimal PWL solution $z^{*}(x)$ to the mp-QP (14) - (15). In order to minimize the real-time computational complexity we require that the state space partition is orthogonal and can be represented as a $k-d$ tree, such that the search complexity is logarithmic with respect to the number of regions. The $k-d$ tree [5] is a hierarchical data structure where a hyperrectangle can be sub-divided into smaller hyper-rectangles allowing the local resolution to be adapted (cf. Figure 1). When searching the tree, only one scalar comparison is required at each level, leading to extremely fast real-time MPC computations.

The main idea of the approximate mp-QP algorithm is to compute the solution of the mp-QP problem (14) - (15) at the $2^{n}$ vertices of a considered hyper-rectangle $X_{0}$ by solving up to $2^{n}$ QPs. Based on these solutions, a feasible local approximation $\hat{z}_{0}(x)$ to the PWL optimal solution $z^{*}(x)$, valid in the whole hyper-rectangle $\boldsymbol{X}_{0}$, is computed by using Lemma 2 given in the previous section. If such an approximation exists, and the maximal cost function approximation error $\varepsilon_{0}$ in $\boldsymbol{X}_{0}$ is smaller than some prescribed tolerance $\bar{\varepsilon}>0$, no further refinement of the hyper-rectangle $\boldsymbol{X}_{0}$ is needed. Otherwise, $\boldsymbol{X}_{0}$ is partitioned into two hyper-rectangles, and the procedure described above is repeated for each of these. In order to reduce the complexity of the partition, the heuristic rule described in [6] is applied when splitting the hyper-rectangle $\boldsymbol{X}_{0}\left(\boldsymbol{X}_{0}\right.$ is divided at the axis along which the change of error is maximal before splitting). The way to compute the error bound $\varepsilon_{0}$ is given in [10].

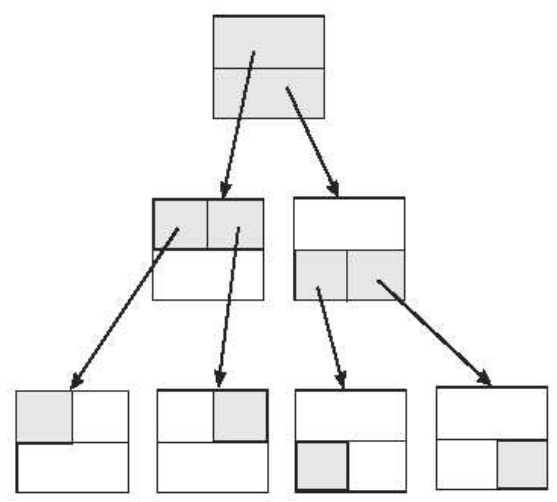

Figure 1: $k-d$ tree partition in a 2-dimensional state space.

\section{Algorithm 1 (approximate mp-QP)}

Step 1. Transform the original mp-QP problem with disturbance input (14) - (15) into the mp-QP problem (20) (21), by applying Lemma 1.

Step 2. Initialize the partition to the whole hyper-rectangle, i.e. $\boldsymbol{P}=\{\boldsymbol{X}\}$. Mark the hyper-rectangle $\boldsymbol{X}$ as unexplored.

Step 3. Select any unexplored hyper-rectangle $\boldsymbol{X}_{0} \in \boldsymbol{P}$. If no such hyper-rectangle exists, go to step 9 .

Step 4. Compute the solution to the QP (20) - (21) for $x$ fixed to each of the $2^{n}$ vertices of the hyper-rectangle $X_{0}$. If all QPs have a feasible solution, go to step 6. Otherwise, go to step 5.

Step 5. Compute the size of $X_{0}$ using some metric. If it is smaller than some given tolerance, mark $\boldsymbol{X}_{0}$ infeasible and explored. Go to step 3. Otherwise, go to step 8 .

Step 6. Compute an affine state feedback $\hat{z}_{0}$ using Lemma 2 , as an approximation to be used in $\boldsymbol{X}_{0}$.

Step 7. Compute the error bound $\varepsilon_{0}$ in $X_{0}$. If $\varepsilon_{0} \leq \bar{\varepsilon}$, mark $\boldsymbol{X}_{0}$ as explored and feasible and go to step 3 .

Step 8. Split the hyper-rectangle $\boldsymbol{X}_{0}$ into two hyperrectangles $\boldsymbol{X}_{1}$ and $\boldsymbol{X}_{2}$ by applying the heuristic rule from [6]. Mark them unexplored, remove $\boldsymbol{X}_{0}$ from $\boldsymbol{P}$, add $\boldsymbol{X}_{1}$ and $\boldsymbol{X}_{2}$ to $\boldsymbol{P}$, and go to step 3 .

Step 9. If necessary, split the hyper-rectangles containing the origin such that $z^{*}(x)=0$ is optimal everywhere in these hyper-rectangles. Terminate. 
This algorithm will terminate with a PWL function that is an approximation to the PWL exact solution and is defined on an inner approximation $\underline{\boldsymbol{X}}_{f}$ of the set $\boldsymbol{X} \cap \boldsymbol{X}_{f}$. The set $\underline{\boldsymbol{X}}_{f}$ is represented as a union of hyper-rectangles.

\section{Illustrative example}

Consider the double integrator:

$$
\boldsymbol{x}(\boldsymbol{t}+1)=\boldsymbol{A x}(\boldsymbol{t})+\boldsymbol{B u}(\boldsymbol{t})+\boldsymbol{T} \boldsymbol{\theta}(\boldsymbol{t})
$$

with:

$$
\boldsymbol{A}=\left[\begin{array}{cc}
1 & \boldsymbol{T}_{s} \\
0 & 1
\end{array}\right], \quad \boldsymbol{B}=\left[\begin{array}{c}
\boldsymbol{T}_{s}^{2} \\
\boldsymbol{T}_{s}
\end{array}\right], \quad \boldsymbol{T}=\left[\begin{array}{ll}
1 & 0 \\
0 & 1
\end{array}\right]
$$

where the sampling interval is $\boldsymbol{T}_{s}=0.05$, and consider the MPC problem with horizon $N=5$, cost matrices $\boldsymbol{Q}=\operatorname{diag}(1,0), \boldsymbol{R}=1$, and the matrix $\boldsymbol{P}>0$ given as the solution of the algebraic Riccati equation. The constraints are:

$$
\begin{aligned}
& -1 \leq \boldsymbol{u} \leq 1 \\
& -0.5 \leq \boldsymbol{x}_{2} \leq 0.5
\end{aligned}
$$

The disturbance vector $\theta=\left[\theta_{1} \theta_{2}\right]^{T}$ has the following bounds:

$$
\begin{aligned}
& -0.01 \leq \theta_{1}(t) \leq 0.01 \\
& -0.015 \leq \theta_{2}(t) \leq 0.015
\end{aligned}
$$

The approximation tolerance $\bar{\varepsilon}>0$ is chosen according to the approach in [10] and it depends on $\boldsymbol{X}_{0}$ such that $\bar{\varepsilon}=\frac{0.175+x_{0}^{T} \Sigma x_{0}}{2}$, where $x_{0}=\arg \min _{x \in X_{0}} x^{T} \Sigma x$.

The state space partition of the robust MPC controller is shown in Figure 2 and it has 126 regions and 12 levels of search. With one scalar comparison required at each level of the $k$ - $d$ tree, 12 arithmetic operations are required in the worst case to determine which region the state belongs to. Totally, 16 arithmetic operations are needed in real-time to compute the control input with this MPC controller (12 comparisons, 2 multiplications and 2 additions).

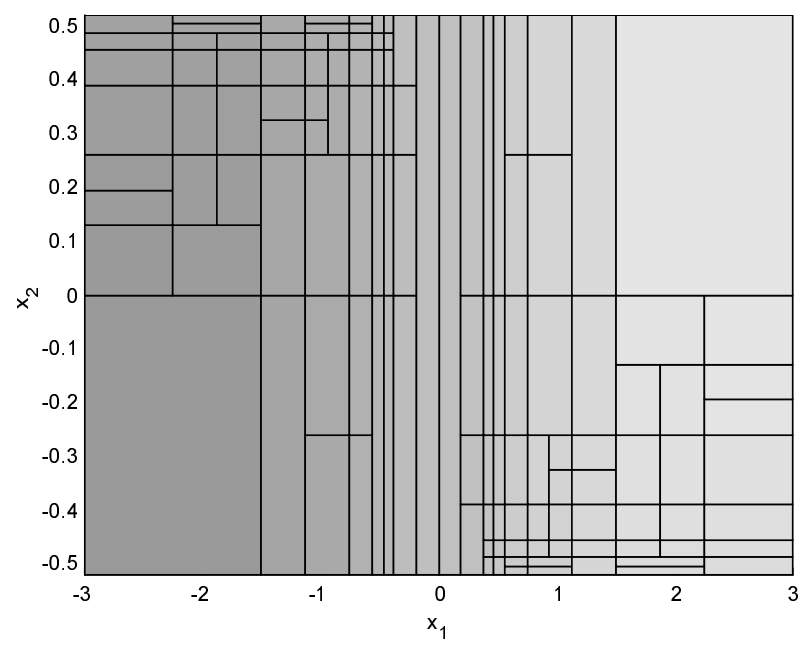

Figure 2: State space partition of the robust MPC with $N=5$.
In Figures 3 and 4, possible realizations for the disturbance variables $\theta_{1}$ and $\theta_{2}$ are given. In Figures 5 to 7 the control and state trajectories obtained with the robust MPC in the presence of disturbance are shown (the solid and dotted curves show the approximate and exact MPC trajectories).

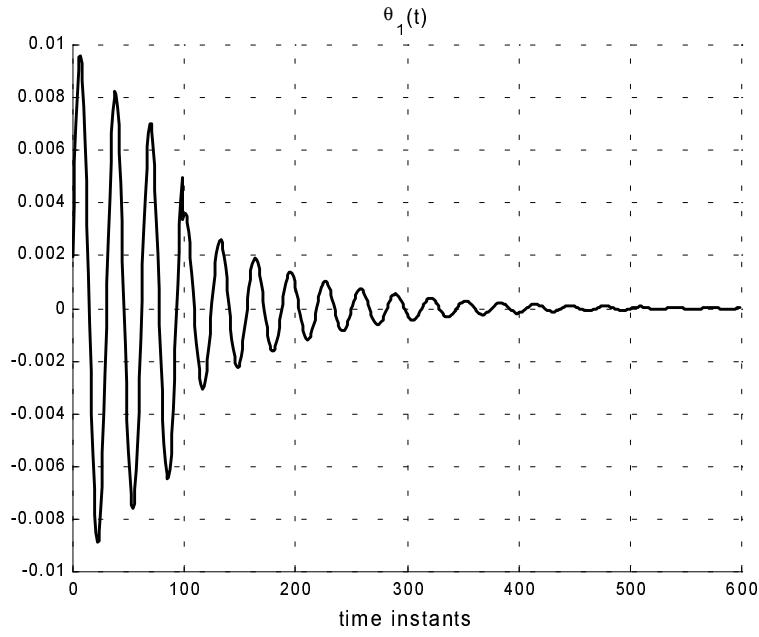

Figure 3: Disturbance $\theta_{1}(t)$.

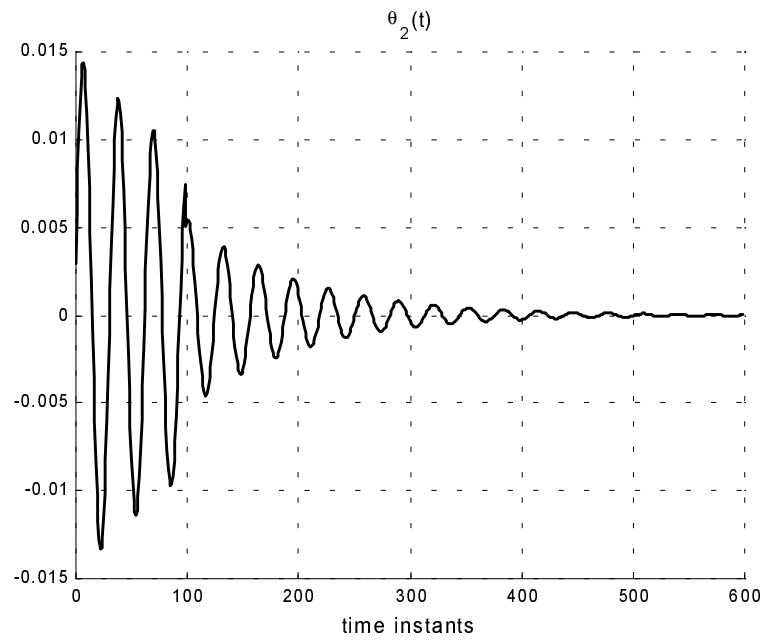

Figure 4: Disturbance $\theta_{2}(t)$.

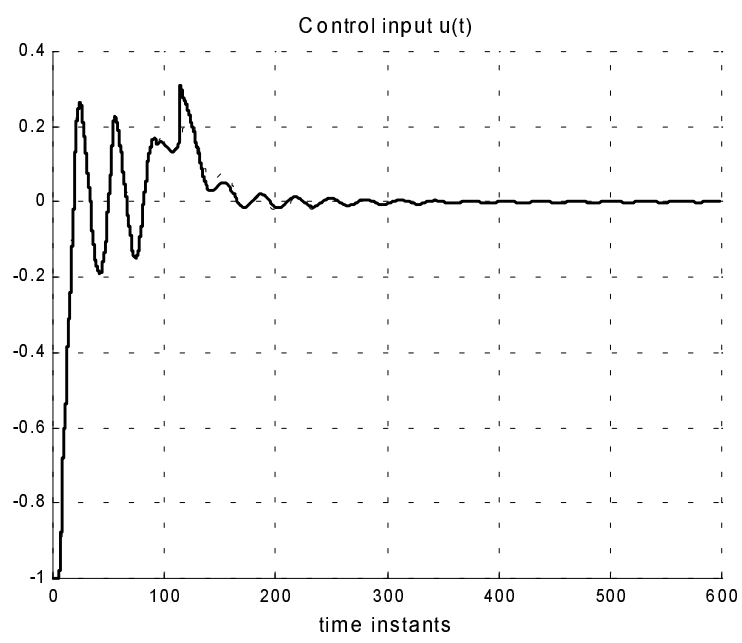

Figure 5: Control input for the robust MPC. 


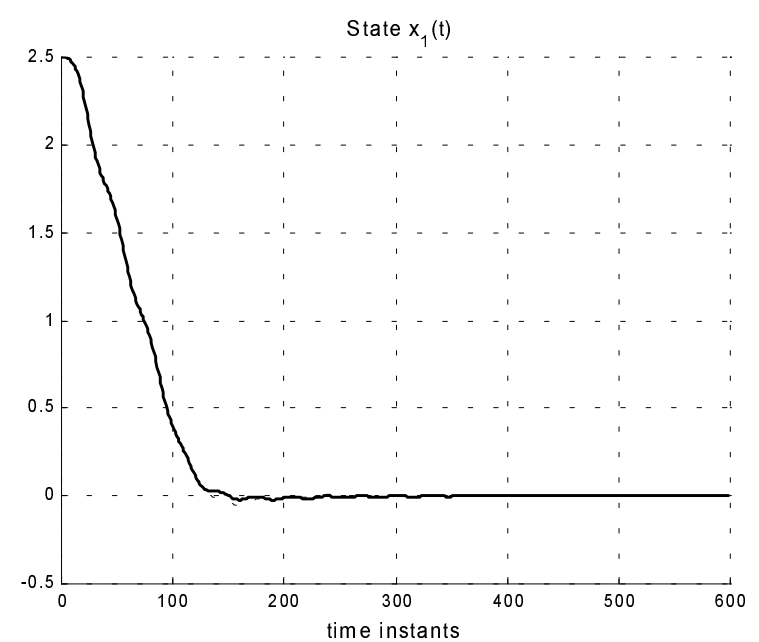

Figure 6: State trajectory $\boldsymbol{x}_{1}$ for the robust MPC.

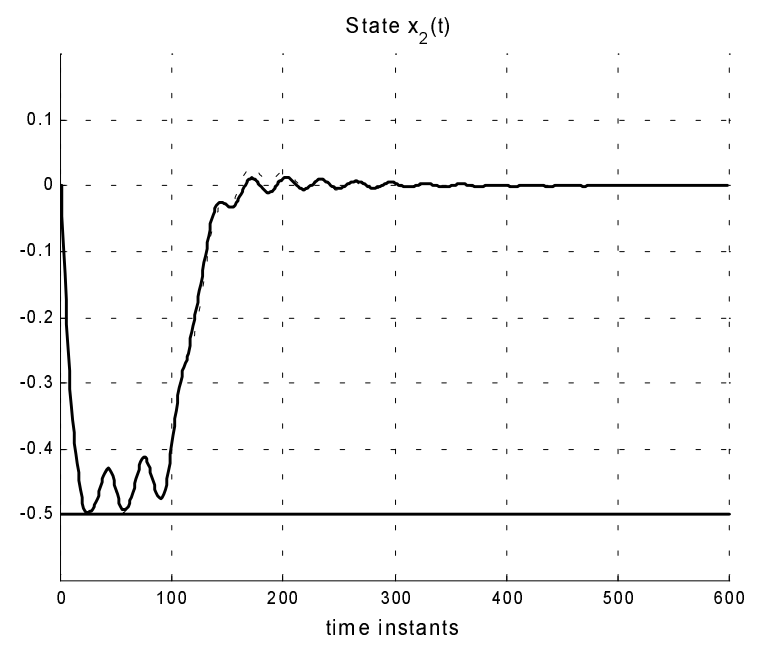

Figure 7: State trajectory $\boldsymbol{x}_{2}$ for the robust MPC.

It can be seen that the robust MPC keeps all constraints imposed on the system.

In Figure 8, the state trajectory $\boldsymbol{x}_{2}$ produced by the nominal MPC in the presence of the same disturbance is shown. The nominal MPC has been designed by assuming that $\theta(t)=0$.

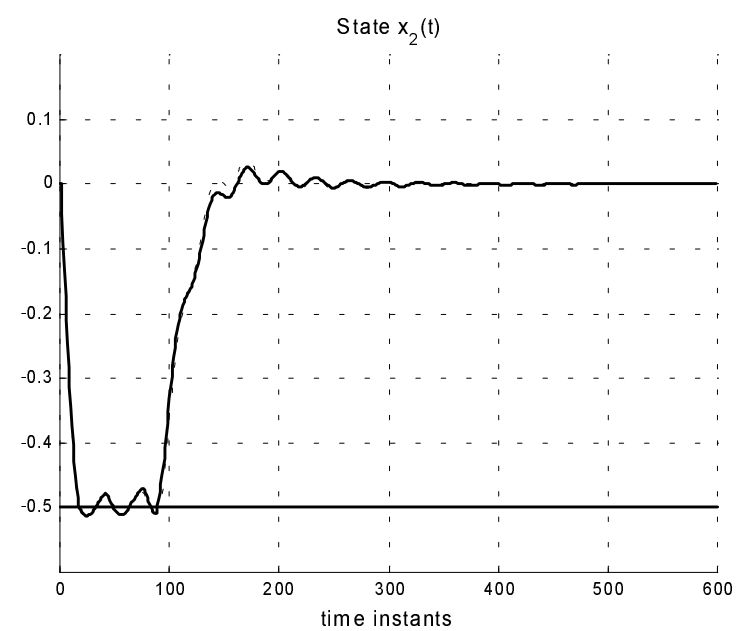

Figure 8: State trajectory $\boldsymbol{x}_{2}$ for the nominal MPC.
It can be seen from Figure 8 that the nominal MPC violates the constraint imposed on state variable $\boldsymbol{x}_{2}$.

\section{Conclusions}

An algorithm for off-line computation of approximate explicit solutions to robust linear constrained MPC problems is described. The resulting explicit piecewise linear state feedback is defined on an orthogonal partition of the state space that allows very efficient real-time computations. It is guaranteed to keep the input and output constraints under all possible disturbance realizations within the specified range.

\section{Acknowledgements}

This work was sponsored by the European Commission through the Research Training Network MAC ("Multi-Agent Control: Probabilistic reasoning, optimal co-ordination, stability analysis and controller design for intelligent hybrid systems", HPRN-CT-1999-00107)

\section{References}

[1]. A. Bemporad, M. Morari, V. Dua, E. N. Pistikopoulos. "The explicit solution of model predictive control via multiparametric quadratic programming", Proc. of American Control Conference, Chicago, pp. 872-876, (2000).

[2]. A. Bemporad, M. Morari, V. Dua, E. N. Pistikopoulos. "The explicit linear quadratic regulator for constrained systems", Automatica, vol. 38, pp. 3-20, (2002).

[3]. A. Bemporad, F. Borrelli, M. Morari. "Robust model predictive control: Piecewise linear explicit solution", Proc. of European Control Conference, Porto, Portugal, pp. 939-944, (2001).

[4]. A. Bemporad, C. Filippi. "Suboptimal explicit MPC via approximate quadratic programming", Proc. of IEEE Conf. Decision and Control, Orlando, pp. 4851-4856, (2001).

[5]. J. L. Bentley. "Multidimensional binary search trees used for associative searching", Communications of the $A C M$, vol. 18, pp. 509-517, (1975).

[6]. A. Grancharova, T. A. Johansen. "Approximate explicit model predictive control incorporating heuristics", Proc. of IEEE International Symposium on Computer Aided Control System Design, Glasgow, Scotland, U.K., pp. 92-97, (2002).

[7]. I. E. Grossmann, K. P. Halemane, R. E. Swaney. "Optimization strategies for flexible chemical processes", Computers \& Chemical Engineering, vol. 7, pp. 439-462, (1983).

[8]. T. A. Johansen, I. Petersen, O. Slupphaug. "Explicit sub-optimal linear quadratic regulation with state and input constraints", Automatica, vol. 38, pp. 1099-1111, (2002).

[9]. T. A. Johansen, A. Grancharova. "Approximate explicit model predictive control implemented via orthogonal search tree partitioning", Proc. of 15-th IFAC World 
Congress, Barcelona, Spain, session T-We-M17, (2002).

[10]. T. A. Johansen, A. Grancharova. "Approximate explicit constrained linear model predictive control via orthogonal search tree", IEEE Trans. Automatic Control, vol. 48, pp. 810-815, (2003).

[11]. N. M. P. Kakalis, V. Dua, V. Sakizlis, J. D. Perkins, E. N. Pistikopoulos. "A parametric optimisation approach for robust MPC", Proc. of 15-th IFAC World Congress, Barcelona, Spain, (2002).

[12]. E. C. Kerrigan, D. Q. Mayne. 'Optimal control of constrained, piecewise affine systems with bounded disturbances", Proc. of IEEE Conf. Decision and Control, Las Vegas, Nevada USA, pp. WeA12-2, (2002)

[13]. V. Sakizlis, N. M. P. Kakalis, V. Dua, J. D. Perkins, E. N. Pistikopoulos. 'Design of robust model-based tracking controllers via parametric programming", Proc. of IEEE International Symposium on Computer Aided Control System Design, Glasgow, Scotland, U.K., pp. 151-156, (2002).

[14]. M. Seron, J. A. De Dona, G. C. Goodwin. 'Global analytical model predictive control with input constraints", Proc. of IEEE Conf. Decision and Control, Sydney, pp. TuA05-2, (2000).

[15]. P. Tøndel, T. A. Johansen, A. Bemporad. "An algorithm for multi-parametric quadratic programming and explicit MPC solutions", Proc. of IEEE Conf. Decision and Control, Orlando, pp. TuP11-4, (2001). 